\title{
Effect of Low Complement C4 on Clinical Characteristics of Patients with First-Episode Neuromyelitis Optica Spectrum Disorder
}

\author{
Chunyang Pan \\ Yi Zhao \\ Haojie Xie \\ Yongyan Zhou \\ Ranran Duan \\ Yanfei Li \\ Yanjie Jia \\ Tao Peng
}

Department of Neurology, The First Affiliated Hospital of Zhengzhou University, Zhengzhou, People's Republic of China
Correspondence: Tao Peng Department of Neurology, The First Affiliated Hospital of Zhengzhou University, Erqi District, Zhengzhou, Henan Province, People's Republic of China

Tel +86I 3673665072

Email zdyfy0420@I63.com
Purpose: To describe and compare the clinical features of patients with first-episode neuromyelitis optica spectrum disorder (NMOSD) in a normal complement $\mathrm{C} 4$ group and a low complement $\mathrm{C} 4$ group, and explore the mechanism by which low complement $\mathrm{C} 4$ affects the clinical features of patients with NMOSD.

Patients and Methods: We retrospectively analyzed clinical data of 169 aquaporin-4 (AQP4) antibody positive patients with NMOSD from the First Affiliated Hospital of Zhengzhou University from December 2013 to March 2021. Prior to treatment, the blood was drawn for detection, and the patients underwent a 3.0 Tesla MRI examination. A low complement $\mathrm{C} 4$ level was defined as a serum complement $\mathrm{C} 4$ level $<0.14 \mathrm{~g} / \mathrm{L}$. Depending on whether the complement $\mathrm{C} 4$ level was reduced, it was divided into the normal complement $\mathrm{C} 4$ group and low complement $\mathrm{C} 4$ group. The basic demographics, clinical manifestations, laboratory examinations, and imaging findings of the two groups were compared.

Results: Among the 169 AQP4 antibody positive patients, 54 were low-complement C4 patients and 115 were normal. There were no significant differences in the demographics, clinical manifestations, treatment options, or admission Expanded Disability Status Scale (EDSS) score between two groups $(\mathrm{P}>0.05)$. The median of discharged EDSS was the same (4 vs 4 ), but the difference between the two was statistically significant $(\mathrm{P}=0.019)$. Compared with the normal complement C4 group, the blood uric acid level (225 vs 179; $\mathrm{P}=0.001)$ and the $\mathrm{C} 3$ level $(1.06$ vs $0.87, \mathrm{P}=0.000)$ of the low complement $\mathrm{C} 4$ group were significantly lower. The incidence of brainstem lesions in patients with low complement $\mathrm{C} 4$ was higher $(53.7 \%$ vs $33 \%, \mathrm{P}=0.01)$.

Conclusion: The treatment effect of the first-episode AQP4 antibody positive NMOSD low complement $\mathrm{C} 4$ group was poor, the blood-brain barrier was more severely damaged, and the disease changes were likely to involve the brainstem.

Keywords: blood-brain barrier, brainstem, humoral immunity, optic neuritis

\section{Introduction}

Neuromyelitis optica spectrum disease (NMOSD) is an inflammatory demyelinating disease of the central nervous system, which is mediated by humoral immunity, most commonly affecting the optic nerve and spinal cord. ${ }^{1,2}$ The clinical manifestations of NMOSD are mostly optic neuritis and long-segment transverse myelitis. It is generally believed that the aquaporin-4 (AQP-4) antibody is the specific disease marker of NMOSD. ${ }^{1-4}$ B cells are the main participants in humoral immunity, and the AQP4-IgG antibody produced after activation into plasma cells binds to the AQP-4 antigen on the surface of astrocytes, ultimately leading to central 
demyelination and neuronal degeneration. ${ }^{5}$ In this process, complement-dependent cytotoxicity and classical complement activation pathways are key parts of the pathogenesis of NMOSD. ${ }^{6-8}$ Studies have shown that the levels of complement $\mathrm{C} 3$ and $\mathrm{C} 4$ in patients with NMOSD are lower than those in healthy individuals. ${ }^{9}$ In clinical practice, we found that the levels of complement $\mathrm{C} 4$ in some patients with NMOSD was not abnormal. Thus, in this study, we aimed to assess the effects of complement $\mathrm{C} 4$ on the clinical characteristics of patients with NMOSD and to determine possible underlying mechanisms to help inform diagnosis and treatment.

\section{Materials and Methods Study Design and Population}

This is a retrospective, observational, single-center study in which we analyzed the clinical data of patients with newly diagnosed NMOSD at the First Affiliated Hospital of Zhengzhou University from December 2013 to March 2021. The inclusion criteria were as follows: (1) first-episode patients; (2) a complete complement test; (3) AQP4 antibody positive patients; (4) without systemic lupus erythematosus (SLE), Sjogren's syndrome, immune complex glomerulonephritis and other factors that affect C4 levels; and (5) subjects did not take drugs that affect uric acid levels before admission. A total of 169 hospitalized patients with NMOSD were selected. All patients met the international NMOSD diagnostic criteria in $2015 .{ }^{4}$ As a retrospective study using anonymized data, this Ethics Committee of Zhengzhou University waived the need for informed consent. The study was reviewed and approved by the Ethics Committee of Zhengzhou University (ethics review number:2019-KY-018). We strictly abide by the "Declaration of Helsinki" and anonymized patient data.

\section{Patient Characteristics}

The basic information of 169 patients was collected, including sex, age at first episode, symptoms onset to admission time, length of hospital stay, clinical manifestations, and treatment methods. The Expanded Disability Status Scale (EDSS) score was used to assess disease severity, which was evaluated by two neurologists. The patients were fasted for at least 12 hours after admission, and blood was drawn prior to treatment. All patients underwent 3.0 Tesla magnetic resonance imaging (MRI) examinations. The Neurology Laboratory of the First
Affiliated Hospital of Zhengzhou University used live cells transfected with AQP4 to detect the AQP4 antibody status in serum or cerebrospinal fluid samples. C4 levels were measured using a scattering turbidimetric method (reference value range: $0.14-0.44 \mathrm{~g} / \mathrm{L}$ ). Uric acid (UA) levels were measured through enzyme colorimetry (reference value range: $200-440 \mu \mathrm{mol} / \mathrm{L}$ ). According to the level of complement $\mathrm{C} 4$, patients were divided into a normal complement $\mathrm{C} 4$ group $(\geq 0.14 \mathrm{~g} / \mathrm{L})$ and a low complement $\mathrm{C} 4$ group $(<0.14 \mathrm{~g} / \mathrm{L})$.

\section{Statistical Analysis}

The SPSS Statistical Software (version 26.0, IBM Corp. Armonk: NY) was used for statistical analysis. Mean \pm standard deviation (SD) values were used to describe continuous variables conforming to the normal distribution, and the $t$-test was used for comparison between two groups. The median and interquartile range (IQR) were used to describe non-conformance normally distributed continuous variables, and the Mann-Whitney $U$-test was used to compare between two groups. Frequency and percentage were used to describe categorical variables, and the chi-squared test was used to compare categorical variables between two groups. Differences were considered statistically significant for values of $p<0.05$.

\section{Results}

\section{Basic Information and Clinical Features}

A total of 169 AQP4 antibody positive subjects (49 men and 120 women) were included in this study. The male-tofemale ratio was approximately $1: 2$, and the median age of the first-episode was 42 years. There were 54 patients in the low-complement $\mathrm{C} 4$ group, including 43 females (79.6\%) and 11 males (20.4\%), and 115 patients in the normal-complement $\mathrm{C} 4$ group, including 77 women (67\%) and 38 men (33\%). There was no significant difference in sex between the two groups $(\mathrm{P}>0.05)$. The median age of the low-complement $\mathrm{C} 4$ group was 43.5 years, and the median length of hospitalization was 18 days. The median age of the normal group was 41.5 years, and the median length of hospitalization was 19 days. There was no significant difference between the two $(\mathrm{P}>0.05)$. The median time from symptom onset to hospital admission was 12 days in both groups, and there was no statistically significant difference between the groups. Both the normal complement $\mathrm{C} 4$ group and the low complement $\mathrm{C} 4$ group showed symptoms of the five major clinical syndromes. 
Table I Demographic Information and Clinical Characteristics of First-Episode NMOSD Patients in C4 Low-Level Group and Normal Group

\begin{tabular}{|c|c|c|c|}
\hline Clinical Characteristics & C4 Normal Group & C4 Low-Level Group & $P$-value \\
\hline Patients (n) & 115 & 54 & \\
\hline \multicolumn{4}{|l|}{ Sex, n (\%) } \\
\hline Male & $38(33)$ & II (20.4) & 0.09 \\
\hline Female & $77(67)$ & $43(79.6)$ & \\
\hline Age, years, median(IQR) & $41.5(25-55)$ & $43.5(28-5 I)$ & 0.548 \\
\hline Length of hospital stay, days, median (IQR) & $19(14-26)$ & $18(12-27)$ & 0.626 \\
\hline In the symptoms onset to admission time, days, median (IQR) & $12(6-20)$ & $12(7-24)$ & 0.927 \\
\hline \multicolumn{4}{|l|}{ Clinical syndrome, n (\%) } \\
\hline Optic neuritis & $32(27.8)$ & $15(27.8)$ & 0.995 \\
\hline Acute myelitis & $83(72.2)$ & $39(72.2)$ & 0.995 \\
\hline Last area of medulla oblongata syndrome & $\mathrm{II}(9.6)$ & $9(16.7)$ & 0.183 \\
\hline Acute brain stem syndrome & $19(16.5)$ & $7(13)$ & 0.55 \\
\hline Acute inter-brain syndrome & $7(6.1)$ & $2(3.7)$ & 0.783 \\
\hline Brain syndrome & $17(14.8)$ & II (20.4) & 0.362 \\
\hline
\end{tabular}

Notes: Statistical tests: Non-normally distributed continuous variables were presented as median (IQR $=25$ th-75th percentile), and categorical variables were described as percentages; The Mann-Whitney $U$-test was used for non-normally distributed measurement data, and the chi-square test was used for categorical variables. Abbreviations: NMOSD, neuromyelitis optica spectrum disorder.

Acute myelitis was the most common condition. There was no statistically significant difference between the two groups in the five major clinical syndromes $(\mathrm{P}>0.05)$ (Table 1).

\section{Cerebrospinal Fluid and Imaging Examinations}

There was no significant difference between the two groups in cerebrospinal fluid (CSF) laboratory tests for white blood cell count, lymphocyte percentage, protein positive rate, or serum albumin $(\mathrm{P}>0.05$ for each measurement). However, serum immunoglobulin in the low complement $\mathrm{C} 4$ group was higher than that in the normal group (12.3 vs $10.5 ; \mathrm{P}=0.029$ ). Based on anatomically informed subdivisions, we summarized the abnormal imaging findings of patients with optic neuromyelitis into five major parts: optic nerve, spinal cord, brainstem, diencephalon, and cerebrum. Twenty-nine (53.7\%) patients in the low complement $\mathrm{C} 4$ group showed brainstem involvement, and $38(33 \%)$ patients in the normal group showed brainstem involvement. A statistically significant difference was found between the number of cases involving the brainstem between the two groups $(33 \%$ vs $53.7 \% ; \mathrm{P}=0.01)$, though there was no statistically significant differences in the incidence of involvement for the remaining four anatomical areas $(\mathrm{P}>0.05)$ (Table 2). The clinical manifestations of NMOSD were divided into last zone syndrome and acute brainstem syndrome, and based on anatomical considerations, we collectively referred to last zone syndrome and acute brainstem syndrome as brainstem syndrome, which is convenient for statistical analyses. Of the 132 patients who did not show brainstem syndrome, 37 (28\%) had brainstem abnormalities on imaging findings. Among the 37 patients with brainstem syndrome, 7 (6.9\%) had negative brainstem imaging findings (Table 3 ).

\section{Disease Severity and Treatment Plan}

There was no significant difference between the normal complement $\mathrm{C} 4$ group and the low complement $\mathrm{C} 4$ group in admission EDSS scores $(\mathrm{P}>0.05)$, but the difference in the discharge EDSS scores was statistically significant (4 vs 4; $\mathrm{P}=0.019$ ). The median admission EDSS score was 5 for the normal complement $\mathrm{C} 4$ group and the low complement $\mathrm{C} 4$ group; the median of the discharged EDSS score was 4. During the acute phase of hospitalization, there was no statistically significant difference between the two groups in the use of one immunotherapy regimen alone or in combination with two or more immunotherapy regimens $(\mathrm{P}>0.05)$ (Table 4).

\section{Clinicopathological Findings}

Compared with the normal complement $\mathrm{C} 4$ group, routine blood values (white blood cell, red blood cell, haemoglobin, platelet, neutrophil, lymphocyte, monocyte count, neutrophil- 
Table 2 Results of Cerebrospinal Fluid and MRI Examinations in First-Episode NMOSD Patients in the Low-Level and Normal Groups of $\mathrm{C} 4$

\begin{tabular}{|c|c|c|c|}
\hline Clinical Characteristics & C4 Normal Group & C4 Low-Level Group & $P$-value \\
\hline Patients (n) & 115 & 54 & \\
\hline \multicolumn{4}{|l|}{ CSF } \\
\hline WBC, median (IQR), (106/L) & $7(2-34)$ & $4(2-26)$ & 0.821 \\
\hline Lymphocyte, median (IQR), (\%) & $75.5(68-84)$ & $76(70-81)$ & 0.785 \\
\hline Protein-positive rates, n (\%) & $3 I(35.6)$ & 16(39) & 0.71 \\
\hline \multicolumn{4}{|l|}{ Immunoglobulin, median (IQR) } \\
\hline CSF (mg/L) & $40.3(23.4-66.0)$ & $45.7(29.2-82.1)$ & 0.091 \\
\hline $\operatorname{Serum}(g / L)$ & $10.5(8.7-12.3)$ & $12.3(9.5-17.1)$ & $0.029 *$ \\
\hline \multicolumn{4}{|l|}{ Albumin, median (IQR) } \\
\hline CSF (mg/L) & $223.4(152.4-345.7)$ & $247.8(I 37.3-400.3)$ & 0.87 \\
\hline $\operatorname{Serum}(g / L)$ & $41.5(39.3-44.7)$ & $40.8(38.7-45.5)$ & 0.561 \\
\hline \multicolumn{4}{|l|}{ MRI, n (\%) } \\
\hline Optic nerve & $6(5.2)$ & $3(5.6)$ & I \\
\hline Spinal cord & $92(80)$ & $4 I(77.4)$ & 0.695 \\
\hline Brain stem & $38(33)$ & $29(53.7)$ & $0.01 *$ \\
\hline Diencephalon & $5(4.3)$ & $6(11.3)$ & 0.173 \\
\hline Cerebrum & $20(17.4)$ & I5(27.8) & 0.12 \\
\hline
\end{tabular}

Notes: $* P<0.05$; Statistical tests: Non-normally distributed continuous variables were presented as median (IQR $=25$ th-75th percentile), and categorical variables were described as percentages; The Mann-Whitney $U$-test was used for non-normally distributed measurement data, and the chi-square test was used for categorical variables. Abbreviations: NMOSD, neuromyelitis optica spectrum disorder; CSF, cerebrospinal fluid; WBC, white blood cell; MRI, magnetic resonance imaging.

Table 3 Brain Stem Syndrome and MRI Brain Stem Lesions

\begin{tabular}{|l|l|l|l|}
\hline \multirow{2}{*}{ Brain Stem Lesions } & \multicolumn{2}{|l|}{ Brain Stem Syndrome } & \multirow{2}{*}{ Total } \\
\cline { 2 - 3 } & Negative & Positive & \\
\hline Negative & 95 & 7 & 102 \\
Positive & 37 & 30 & 67 \\
Total & 132 & 37 & 169 \\
\hline
\end{tabular}

Abbreviation: MRI, magnetic resonance imaging.

to-lymphocyte ratio, lymphocyte-to-monocyte ratio, and lymphocyte-to-monocyte ratio) of the low complement $\mathrm{C} 4$ group were not statistically different $(\mathrm{P}>0.05)$, but the difference in eosinophils between the two groups was statistically significant ( 0.02 vs $0.07 ; \mathrm{P}<0.001$ ). The low complement $\mathrm{C} 4$ group showed a lower complement $\mathrm{C} 3$ level than the normal complement $\mathrm{C} 4$ group, and the difference between the two groups was statistically significant $(0.87$ vs $1.06 ; \mathrm{P}<0.001)$. UA level in the low complement $\mathrm{C} 4$ group was lower than that in the normal group (179 vs 225; $\mathrm{P}=0.001$ ) (Table 5).

\section{Discussion}

NMOSD represents a group of inflammatory demyelinating diseases of the central system that is mainly mediated by humoral immunity, in which the complement also plays
Table 4 Severity of Disease and Treatment Options in FirstEpisode NMOSD Patients in the Low-Level and Normal Groups of $\mathrm{C} 4$

\begin{tabular}{|l|l|l|l|}
\hline $\begin{array}{l}\text { Clinical } \\
\text { Characteristics }\end{array}$ & $\begin{array}{l}\text { C4 Normal } \\
\text { Group }\end{array}$ & $\begin{array}{l}\text { C4 Low-Level } \\
\text { Group }\end{array}$ & P-value \\
\hline Patients (n) & 115 & 54 & \\
\hline $\begin{array}{l}\text { EDSS, median } \\
\text { (IQR) }\end{array}$ & & & \\
Initial & $5(3-6)$ & $5(4-7)$ & 0.099 \\
Discharge & $4(2-5)$ & $4(3-6)$ & $0.019^{*}$ \\
\hline $\begin{array}{l}\text { The acute } \\
\text { treatment, n (\%) } \\
\text { One drug alone } \\
\text { Combination } \\
\text { medication }\end{array}$ & $97(84.3)$ & $46(85.2)$ & \\
\hline
\end{tabular}

Notes: $* P<0.05$; Statistical tests: Non-normally distributed continuous variables were presented as median $(\mathrm{IQR}=25$ th -75 th percentile $)$, and categorical variables were described as percentages; The Mann-Whitney U-test was used for nonnormally distributed measurement data, and the chi-square test was used for categorical variables.

Abbreviations: NMOSD, neuromyelitis optica spectrum disorder; EDSS, Expanded Disability Status Scale.

a vital role. ${ }^{5-8}$ Complement assays in clinical laboratories include the detection of $\mathrm{C} 3$ and $\mathrm{C} 4$ levels. We found that grouping by $\mathrm{C} 3$ levels, the main observation indicators between the groups were not statistically significant. In 
Table 5 Laboratory Test Results of First-Episode NMOSD Patients in C4 Low-Level Group and Normal Group

\begin{tabular}{|c|c|c|c|}
\hline Clinical Characteristics & C4 Normal Group & C4 Low-Level Group & $P$-value \\
\hline Patients (n) & 115 & 54 & \\
\hline \multicolumn{4}{|l|}{ Blood routine } \\
\hline WBC, median (IQR), $\left(10^{9} / \mathrm{L}\right)$ & $6.7(5.2-9)$ & $6.2(4.4-8.8)$ & 0.143 \\
\hline $\mathrm{RBC}, \mathrm{x} \pm \mathrm{s},\left(10^{12} / \mathrm{L}\right)$ & $4.3 \pm 0.46$ & $4.21 \pm 0.65$ & 0.298 \\
\hline $\mathrm{Hb}, \mathrm{x} \pm \mathrm{s},(\mathrm{g} / \mathrm{L})$ & $|27.17 \pm 1.3|$ & $124.85 \pm 1.95$ & 0.323 \\
\hline PLT, median (IQR), (10\%/L) & $225(183-274)$ & $215(179-250)$ & 0.201 \\
\hline Neutrophils, median (IQR), (109/L) & $4.50(3.13-6.10)$ & $4.01(2.93-5.82)$ & 0.382 \\
\hline Lymphocyte, median (IQR), (10\%/L) & $1.64(1.20-2.04)$ & $1.26(1.05-2.02)$ & 0.144 \\
\hline Monocytes, median (IQR), (109/L) & $0.49(0.36-0.61)$ & $0.44(0.29-0.60)$ & 0.199 \\
\hline Eosinophils, median (IQR), (109/L) & $0.07(0.03-0.14)$ & $0.02(0.01-0.03)$ & $<0.00 I^{*}$ \\
\hline NLR, median (IQR) & $2.6 I(1.78-4.50)$ & $2.92(1.66-4.69)$ & 0.832 \\
\hline LMR, median (IQR) & $3.80(2.40-5.08)$ & $3.93(2.45-5.23)$ & 0.891 \\
\hline NMR, median (IQR) & $9.58(7.08-11.74)$ & $8.52(6.67-16.65)$ & 0.927 \\
\hline C3, median (IQR), (g/L) & $1.06(0.94-1.20)$ & $0.87(0.73-1.06)$ & $<0.001 *$ \\
\hline \multicolumn{4}{|l|}{ Renal function } \\
\hline Urea, median (IQR), (mmol/L) & $4.50(3.58-5.75)$ & $4.20(3.25-5.48)$ & 0.505 \\
\hline $\mathrm{Cr}, \operatorname{median}(\mathrm{IQR}),(\mu \mathrm{mol} / \mathrm{L})$ & $53(46-6 I)$ & $53(47-58)$ & 0.854 \\
\hline Uric acid, median(IQR), ( $\mu \mathrm{mol} / \mathrm{L})$ & $225(177-287)$ & $179(\mid 58-225)$ & $0.001 *$ \\
\hline
\end{tabular}

Notes: $* P<0.05$; Statistical tests: Continuous variables were presented as mean \pm SD or median (IQR $=25$ th-75th percentile; The $t$-test was used for normally distributed measurement data, and The Mann-Whitney U-test was used for non-normally distributed measurement data.

Abbreviations: NMOSD, neuromyelitis optica spectrum disorder; WBC, white blood cell; RBC, red blood cell; Hb, hemoglobin; PLT, platelet; NLR, neutrophil-tolymphocyte ratio; LMR, lymphocyte to monocyte ratio; NMR, neutrophil to monocyte ratio; Cr, Creatinine.

this study, we searched the literatures and found that the research related to $\mathrm{C} 4$ used the reference range of $\mathrm{C} 4$ level of the respective laboratory, ${ }^{10-12}$ so we also used the reference range of the laboratory of our hospital to determine the grouping.

A total of 169 subjects were included in this study, and the male-to-female ratio was approximately 1:2, which was significantly higher than the results of a global NMOSD epidemiological survey. ${ }^{13}$ Various studies have confirmed that the complement system is involved in autoimmune diseases, such as Sjogren's syndrome $^{14}$ and SLE. ${ }^{15}$ Sjogren's syndrome and SLE are more common in female patients, ${ }^{16-18}$ and NMOSD is often comorbid with the above-mentioned autoimmune diseases. The high male-to-female ratio in this study may be related to the elimination of patients with autoimmune diseases, such as Sjogren's syndrome and systemic lupus erythematosus. The median age of the patients included in this study was 42 years, which is consistent with the results of a global NMOSD epidemiological survey. ${ }^{13}$ Of the 169 subjects, 122 (76.3\%) had clinical manifestations of acute myelitis, indicating that the spinal cord was the most common site of involvement. However, there was no significant difference in clinical manifestations between the two groups. It is speculated that complement abnormalities have no significant impact on clinical manifestations.

We summarized the abnormal imaging findings of NMOSD patients into five major regions: optic nerve, spinal cord, brain stem, diencephalon, and cerebrum, according to anatomical structure. By comparing the groups, we found that the difference between the two groups in the brainstem was statistically significant $(\mathrm{P}=0.01)$, suggesting that the brainstem is easily involved in patients with abnormal complement levels. We also compared the imaging findings with the clinical syndromes and found that approximately $28 \%$ of brainstem syndrome-negative patients showed brainstem lesions on imaging, and approximately $18.9 \%$ of brainstem syndrome-positive patients showed no signs of brainstem lesions. This indicated that, if conditions permit, it is necessary for clinicians to perform head MRI examinations, which may reveal more "invisible" lesions. However, the resolution limitations of MRI scanning may also lead to no remarkable observations. In such a case, the patient needs to be reviewed again, the medical history should be re-assessed, and evidence for brainstem syndrome must be investigated. 
AQP-4 is highly expressed in the foot processes of astrocytes adjacent to the nerve vasculature and is particularly highly expressed in the brainstem, optic nerve, spinal cord, and periventricular regions. ${ }^{19}$ The complement cascade can be activated through classical, alternative, or lectin pathways. ${ }^{20}$ The AQP-4-IgG antibody binds to the antigen in the brainstem to form a large number of antigen-antibody complexes, and $\mathrm{Clq}$ recognizes the antibody-antigen complex to activate the classical pathway. ${ }^{20,21} \mathrm{C} 1 \mathrm{q}$ cleaves $\mathrm{C} 1 \mathrm{r}$ and activates the $\mathrm{C} 1 \mathrm{~s}$ protease to form the $\mathrm{C} 1$ complex (ie, $\mathrm{C} 1 \mathrm{qC} 1 \mathrm{r} 2 \mathrm{C} 1 \mathrm{~s} 2$ ); $\mathrm{C} 4$ and $\mathrm{C} 2$ are then cleaved to form the classical pathway $\mathrm{C} 3$ convertase $(\mathrm{C} 4 \mathrm{bC} 2 \mathrm{a})$, and the enzymatically active $\mathrm{C} 4 \mathrm{bC} 2 \mathrm{a}$ cleaves $\mathrm{C} 3$ to form two fragments of $\mathrm{C} 3 \mathrm{a}$ and $\mathrm{C} 3 \mathrm{~b}, \mathrm{C} 3 \mathrm{~b}$ combined with $\mathrm{C} 4 \mathrm{~b} 2 \mathrm{a}$ to produce $\mathrm{C} 4 \mathrm{~b} 2 \mathrm{aC} 3 \mathrm{~b}$, and $\mathrm{C} 5$ convertase continues to activate downstream complement, finally completing the complement cascade reaction. ${ }^{20}$ In this process, $\mathrm{C} 4$ is consumed; as such, we observed a decrease in serum complement $\mathrm{C} 4$ levels in clinical practice. However, some patients with NMOSD have normal complement $\mathrm{C} 4$ levels. By comparing the $\mathrm{C} 3$ levels of the two groups, it was found that low complement $\mathrm{C} 4$ was accompanied by low complement $\mathrm{C} 3$, and the $\mathrm{C} 3$ level of the normal complement $\mathrm{C} 4$ group was normal. As such, we speculate that not all patients with NMOSD have an activated complement system. Previous studies have shown that the brainstem is an important component of NMOSD, and a retrospective analysis showed that the incidence of brainstem lesions in NMOSD patients with serum AQP-4 antibody positivity was significantly higher than those with AQP-4 negativity, and that the recurrence rate was higher as well. ${ }^{22}$ The brainstem maintains human breathing and heartbeat. The brainstem of low-complement $\mathrm{C} 4$ patients is more vulnerable than that of the normal complement group patients, indicating that these patients are at a high risk of respiratory and cardiac arrest, and that the condition is more dangerous. Clinicians should very carefully monitor these patients and identify signs of imminent danger over time.

We evaluated the EDSS scores of each patient on admission and discharge. The median values of the discharged EDSS scores of the two groups were the same, and the differences were statistically significant, suggesting that patients with low complement $\mathrm{C} 4$ had a poor prognosis. However, there was no significant difference in the treatment option. A total of $85.2 \%$ of patients with low complement $\mathrm{C} 4$ levels were treated with a single immunosuppressive method. The poor treatment effect of the $\mathrm{C} 4$ group with low complement may be related to the single treatment regimen.

This study found that the eosinophil count in the low complement $\mathrm{C} 4$ group was significantly lower than that in the normal complement group. Previous studies have shown that $\mathrm{C} 3 \mathrm{a}$ can indirectly stimulate neutrophils by activating eosinophils, while $\mathrm{C} 5 \mathrm{a}$ is a chemotactic agent for both neutrophils and eosinophils. ${ }^{23}$ After the classical complement activation pathway is activated, a large amount of $\mathrm{C} 3 \mathrm{a}$ and $\mathrm{C} 5 \mathrm{a}$ are produced at the lesion site, then eosinophils in the chemotactic serum cross the bloodbrain barrier (BBB) and indirectly activate neutrophils. ${ }^{24}$ After degranulation of granulocytes, the release of hydrolytic granzymes, bioactive lipids and superoxide anions may incur brain damage. ${ }^{24}$ A study showed that the transgene expression of $\mathrm{C} 3 \mathrm{a}$ in the central nervous system significantly exacerbated experimental autoimmune encephalomyelitis and caused inflammatory cell infiltration around blood vessels. ${ }^{25}$ These results indicate that eosinophils play a key role in the pathogenesis of NMOSD.

UA, the end product of purine metabolism, is a natural water-soluble antioxidant that accounts for $60 \%$ of the free radical-scavenging activity in human blood, and it is a known scavenger of peroxynitrite (PN), also can be modified by drugs and diet. ${ }^{26-30}$ Nitrogen oxide (NO) and its toxic metabolite $\mathrm{PN}$ are key mediators of nerve damage in Parkinson's disease. ${ }^{31} \mathrm{PN}$ is involved in the pathogenesis of central nervous system inflammatory diseases $^{26}$ and can cause DNA cleavage, lipid peroxidation, and oxidation and nitrification of amino acid residues and guanine. Hooper et $\mathrm{al}^{26}$ proposed that UA may protect the permeability of the BBB by directly clearing PN. Another study showed that UA exerts its neuroprotective effect by eliminating the oxidative toxicity of PN and its downstream free radicals. ${ }^{32}$ The plasma UA level is 10 times that of the CSF content and the two are positively correlated, indicating that UA is produced in the periphery and its penetration of the brain is restricted by the BBB. In other words, plasma UA and the integrity of the BBB jointly determine the CSF UA level. ${ }^{33}$ Serum $\mathrm{UA}$ protects the integrity of the $\mathrm{BBB}$, reduces its permeability, and can also reduce the infiltration of inflammatory cells, thereby reducing brain inflammation. ${ }^{34}$ Previous studies have shown reduced serum UA levels in patients with NMOSD. ${ }^{35}$ In this study, the blood UA level of the low complement $\mathrm{C} 4$ group was lower than that of the normal complement $\mathrm{C} 4$ group, indicating that the BBB damage and the infiltration of inflammatory cells 
in patients with low complement levels were more serious.

With the in-depth exploration of the mechanism of NMOSD by researchers, people have reported novel markers for disease prediction, such as serum glial fibrillary acidic protein (sGFAP) and serum neurofilament light chain (sNfL). GFAP is mainly expressed by astrocytes and is an intermediate fibrous protein that forms the cytoskeleton of astrocytes. ${ }^{36} \mathrm{NfL}$ is a component of the neurofilament. ${ }^{37}$ Nerve axon damage leads to the release of neurofilament, and then NfL can be detected in the cerebrospinal fluid and blood. ${ }^{38,39}$ sGFAP levels and sNfL levels are closely related to EDSS score and age, and sGFAP levels are also closely related to the occurrence of recent relapse. ${ }^{40,41}$ A multicenter, double-blind, randomized controlled trial showed that serum GFAP can be used as a biomarker of NMOSD activity, risk of disease, and treatment effect. ${ }^{41}$

Our study has certain limitations. First, this was a retrospective study, and therefore inherently biased. In addition, the number of cases included in the study was small. As such, multicenter, large-sample clinical studies are needed to further confirm this study's conclusions.

\section{Conclusion}

In summary, AQP4 antibody-positive patients with low complement $\mathrm{C} 4$ levels have more severe damage to the blood-brain barrier, and changes in the disease easily involve the brainstem. Therefore, head MRI should be performed as frequently as is clinically possible. More multi-center large cohort studies are needed to further confirm.

\section{Disclosure}

The authors report no conflicts of interest in this work.

\section{References}

1. Wingerchuk D, Hogancamp W, O'Brien P, Weinshenker B. The clinical course of neuromyelitis optica (Devic's syndrome). Neurology. 1999;53(5):1107-1114. doi:10.1212/WNL.53.5.1107

2. Wingerchuk D, Lennon V, Pittock S, Lucchinetti C, Weinshenker B. Revised diagnostic criteria for neuromyelitis optica. Neurology. 2006;66(10):1485-1489. doi:10.1212/01.wnl.0000216139.44259.74

3. Lennon V, Kryzer T, Pittock S, Verkman A, Hinson S. IgG marker of optic-spinal multiple sclerosis binds to the aquaporin-4 water channel. J Exp Med. 2005;202(4):473-477. doi:10.1084/jem.20050304

4. Wingerchuk D, Banwell B, Bennett $\mathrm{J}$, et al. International consensus diagnostic criteria for neuromyelitis optica spectrum disorders. Neurology. 2015;85(2):177-189. doi:10.1212/WNL.00000000000 01729
5. Jacob A, McKeon A, Nakashima I, et al. Current concept of neuromyelitis optica (NMO) and NMO spectrum disorders. J Neurol Neurosurg Psychiatry. 2013;84(8):922-930. doi:10.1136/jnnp-2012302310

6. Saadoun S, Waters P, Bell B, Vincent A, Verkman A, Papadopoulos M. Intra-cerebral injection of neuromyelitis optica immunoglobulin $\mathrm{G}$ and human complement produces neuromyelitis optica lesions in mice. Brain. 2010;133:349-361. doi:10.1093/brain/ awp309

7. Tradtrantip L, Yao X, Su T, Smith A, Verkman A. Bystander mechanism for complement-initiated early oligodendrocyte injury in neuromyelitis optica. Acta Neuropathol. 2017;134(1):35-44. doi:10.1007/ s00401-017-1734-6

8. Zhang H, Bennett J, Verkman A. Ex vivo spinal cord slice model of neuromyelitis optica reveals novel immunopathogenic mechanisms. Ann Neurol. 2011;70(6):943-954. doi:10.1002/ana.22551

9. Chen Y, Li R, Wu A, Shu Y, Lu Z, Hu X. The complement and immunoglobulin levels in NMO patients. Neurol Sci. 2014;35 (2):215-220. doi:10.1007/s10072-013-1481-y

10. Sise M, Strohbehn I, Chute D, et al. Low complement C4 predicts improvement of kidney function after direct-acting antiviral therapy for hepatitis C virus. Hepatol Commun. 2020;4(8):1206-1217. doi: $10.1002 /$ hep 4.1528

11. Ramsey-Goldman R, Alexander R, Massarotti E, et al. Complement activation in patients with probable systemic lupus erythematosus and ability to predict progression to American college of rheumatology-classified systemic lupus erythematosus. Arthritis Rheumatol. 2020;72(1):78-88. doi:10.1002/art.41093

12. Garabet L, Gilboe I, Mowinckel M, et al. Antiphospholipid antibodies are associated with low levels of complement $\mathrm{C} 3$ and $\mathrm{C} 4$ in patients with systemic lupus erythematosus. Scand J Immunol. 2016;84(2):95-99. doi:10.1111/sji.12445

13. Hor J, Asgari N, Nakashima I, et al. Epidemiology of neuromyelitis optica spectrum disorder and its prevalence and incidence worldwide. Front Neurol. 2020;11:501. doi:10.3389/fneur.2020.00501

14. Ramos-Casals M, Brito-Zerón $\mathrm{P}$, Yagüe $\mathrm{J}$, et al. Hypocomplementaemia as an immunological marker of morbidity and mortality in patients with primary Sjogren's syndrome. Rheumatology. 2005;44(1):89-94. doi:10.1093/rheumatology/keh407

15. Karp D. Complement and systemic lupus erythematosus. Curr Opin Rheumatol. 2005;17(5):538-542. doi:10.1097/01.bor.00001727 99.03379.86

16. Mavragani C, Moutsopoulos H. The geoepidemiology of Sjögren's syndrome. Autoimmun Rev. 2010;9(5):A305-A310. doi:10.1016/j. autrev.2009.11.004

17. Li M, Zhang W, Leng X, et al. Chinese SLE Treatment and Research group (CSTAR) registry: I. Major clinical characteristics of Chinese patients with systemic lupus erythematosus. Lupus. 2013;22 (11):1192-1199. doi:10.1177/0961203313499086

18. Petri M. Epidemiology of systemic lupus erythematosus. Best Pract Res Clin Rheumatol. 2002;16(5):847-858. doi:10.1053/berh.200 2.0259

19. Wingerchuk D. Neuromyelitis optica spectrum disorders. Continuum. 2010;16:105-121.

20. Gavriilaki M, Kimiskidis V, Gavriilaki E. Precision medicine in neurology: the inspirational paradigm of complement therapeutics. Pharmaceuticals. 2020;13(11):341.

21. Brunhouse R, Cebra J. Isotypes of IgG: comparison of the primary structures of three pairs of isotypes which differ in their ability to activate complement. Mol Immunol. 1979;16(11):907-917. doi:10.1016/0161-5890(79)90089-0

22. Asgari N, Skejoe H, Lillevang S, Steenstrup T, Stenager E, Kyvik K. Modifications of longitudinally extensive transverse myelitis and brainstem lesions in the course of neuromyelitis optica (NMO): a population-based, descriptive study. BMC Neurol. 2013;13:33. doi:10.1186/1471-2377-13-33 
23. Daffern P, Pfeifer P, Ember J, Hugli T. C3a is a chemotaxin for human eosinophils but not for neutrophils. I. C3a stimulation of neutrophils is secondary to eosinophil activation. $J$ Exp Med. 1995;181(6):2119-2127. doi:10.1084/jem.181.6.2119

24. Henson P. The immunologic release of constituents from neutrophil leukocytes. II. Mechanisms of release during phagocytosis, and adherence to nonphagocytosable surfaces. J Immunol. 1971;107 (6):1547-1557.

25. Boos L, Campbell I, Ames R, Wetsel R, Barnum S. Deletion of the complement anaphylatoxin $\mathrm{C} 3$ a receptor attenuates, whereas ectopic expression of $\mathrm{C} 3 \mathrm{a}$ in the brain exacerbates, experimental autoimmune encephalomyelitis. J Immunol. 2004;173(7):4708-4714. doi:10.4049/ jimmunol.173.7.4708

26. Hooper D, Scott G, Zborek A, et al. Uric acid, a peroxynitrite scavenger, inhibits CNS inflammation, blood-CNS barrier permeability changes, and tissue damage in a mouse model of multiple sclerosis. FASEB J. 2000;14(5):691-698. doi:10.1096/fasebj.14.5.691

27. Ames B, Cathcart R, Schwiers E, Hochstein P. Uric acid provides an antioxidant defense in humans against oxidant- and radical-caused aging and cancer: a hypothesis. Proc Natl Acad Sci U S A. 1981;78 (11):6858-6862. doi:10.1073/pnas.78.11.6858

28. Choi H, Liu S, Curhan G. Intake of purine-rich foods, protein, and dairy products and relationship to serum levels of uric acid: the Third National Health and Nutrition Examination Survey. Arthritis Rheum. 2005;52(1):283-289. doi:10.1002/art.20761

29. Gao X, Qi L, Qiao N, et al. Intake of added sugar and sugar-sweetened drink and serum uric acid concentration in US men and women. Hypertension. 2007;50(2):306-312. doi:10.1161/ HYPERTENSIONAHA.107.091041

30. Gao X, Chen H, Choi H, Curhan G, Schwarzschild M, Ascherio A. Diet, urate, and Parkinson's disease risk in men. Am J Epidemiol. 2008;167(7):831-838. doi:10.1093/aje/kwm385

31. Kouti L, Noroozian M, Akhondzadeh S, et al. Nitric oxide and peroxynitrite serum levels in Parkinson's disease: correlation of oxidative stress and the severity of the disease. Eur Rev Med Pharmacol Sci. 2013;17(7):964-970.
32. Squadrito G, Cueto R, Splenser A, et al. Reaction of uric acid with peroxynitrite and implications for the mechanism of neuroprotection by uric acid. Arch Biochem Biophys. 2000;376(2):333-337. doi:10.1006/abbi.2000.1721

33. Bowman G, Shannon J, Frei B, Kaye J, Quinn J. Uric acid as a CNS antioxidant. J Alzheimers Dis. 2010;19(4):1331-1336. doi:10.3233/ JAD-2010-1330

34. Li X, Tong Q, Xie D, et al. Low serum uric acid levels in patients with acute central nervous system viral infections. Neuroreport. 2017;28(18):1250-1254. doi:10.1097/WNR.0000000000000908

35. Peng F, Zhong X, Deng X, et al. Serum uric acid levels and neuromyelitis optica. J Neurol. 2010;257(6):1021-1026. doi:10.1007/ s00415-010-5455-1

36. Brenner M, Johnson A, Boespflug-Tanguy O, Rodriguez D, Goldman J, Messing A. Mutations in GFAP, encoding glial fibrillary acidic protein, are associated with Alexander disease. Nat Genet. 2001;27(1):117-120. doi:10.1038/83679

37. Lee M, Cleveland D. Neuronal intermediate filaments. Annu Rev Neurosci. 1996;19:187-217. doi:10.1146/annurev.ne.19.030196.00 1155

38. Petzold A. Neurofilament phosphoforms: surrogate markers for axonal injury, degeneration and loss. J Neurol Sci. 2005;233:183-198. doi:10.1016/j.jns.2005.03.015

39. Bacioglu M, Maia L, Preische O, et al. Neurofilament light chain in blood and CSF as marker of disease progression in mouse models and in neurodegenerative diseases. Neuron. 2016;91(1):56-66. doi:10.1016/j.neuron.2016.05.018

40. Watanabe M, Nakamura Y, Michalak Z, et al. Serum GFAP and neurofilament light as biomarkers of disease activity and disability in NMOSD. Neurology. 2019;93(13):e1299-e1311. doi:10.1212/ WNL.0000000000008160

41. Aktas O, Smith M, Rees W, et al. Serum glial fibrillary acidic protein: a neuromyelitis optica spectrum disorder biomarker. Ann Neurol. 2021;89(5):895-910. doi:10.1002/ana.26067
Neuropsychiatric Disease and Treatment

\section{Publish your work in this journal}

Neuropsychiatric Disease and Treatment is an international, peerreviewed journal of clinical therapeutics and pharmacology focusing on concise rapid reporting of clinical or pre-clinical studies on a range of neuropsychiatric and neurological disorders. This journal is indexed on PubMed Central, the 'PsycINFO' database and CAS, and

\section{Dovepress}

is the official journal of The International Neuropsychiatric Association (INA). The manuscript management system is completely online and includes a very quick and fair peer-review system, which is all easy to use. Visit http://www.dovepress.com/testimonials.php to read real quotes from published authors. 